\title{
BMJ Open Comparative effectiveness and safety of low-strength and high-strength direct oral anticoagulants compared with warfarin: a sequential cohort study
}

\author{
Nicole L Pratt, ${ }^{1}$ Emmae Ramsay, ${ }^{1}$ Lisa M Kalisch Ellett, ${ }^{1}$ Katherine Duszynski, ${ }^{2}$
} Sepehr Shakib, ${ }^{3,4}$ Mhairi Kerr, ${ }^{1}$ Gillian Caughey, ${ }^{3,4}$ Elizabeth Ellen Roughead ${ }^{1}$

To cite: Pratt NL, Ramsay E, Kalisch Ellett LM, et al. Comparative effectiveness and safety of low-strength and high-strength direct oral anticoagulants compared with warfarin: a sequential cohort study. BMJ Open 2019;9:e026486. doi:10.1136/ bmjopen-2018-026486

- Prepublication history and additional material for this paper are available online. To view these files, please visit the journal online (http://dx.doi org/10.1136/bmjopen-2018026486).

Received 13 September 2018 Revised 14 March 2019 Accepted 19 March 2019
Check for updates

(C) Author(s) (or their employer(s)) 2019. Re-use permitted under CC BY-NC. No commercial re-use. See rights and permissions. Published by BMJ.

For numbered affiliations see end of article.

Correspondence to

Dr Nicole L Pratt;

nicole.pratt@unisa.edu.au

\section{ABSTRACT}

Objectives The aim of this study was to compare effectiveness and safety of low-strength and high-strength direct oral anticoagulants (DOACs) with warfarin in the Australian Veteran population.

Design Sequential cohort study using inverse probability of treatment weighting (IPTW) and propensity score matching. Initiators of high-strength (apixaban $5 \mathrm{mg}$, dabigatran $150 \mathrm{mg}$, rivaroxaban $20 \mathrm{mg}$ ) and low-strength DOACS (apixaban $2.5 \mathrm{mg}$, dabigatran $110 \mathrm{mg}$, rivaroxaban $15 \mathrm{mg}$ ) were compared with warfarin initiators.

Setting Australian Government Department of Veterans' Affairs claims database.

Participants 4836 patients who initiated oral anticoagulants $(45.8 \%, 26.0 \%$ and $28.2 \%$ on low-strength high-strength DOACs and warfarin, respectively) between August 2013 and March 2015. Mean age was 85, 75 and 83 years for low-strength, high-strength DOACs and warfarin initiators, respectively.

Main outcome measures One-year risk of hospitalisation for ischaemic stroke, any bleeding event or haemorrhagic stroke. Secondary outcomes were 1-year risk of hospitalisation for myocardial infarction and death. Results Using the IPTW method, no difference in risk of ischaemic stroke or bleeding was found with low-strength DOACs compared with warfarin. As a class, no increased risk of myocardial infarction was found for low-strength DOACs, however, risk was elevated for apixaban (HR $2.25,95 \% \mathrm{Cl} 1.23$ to 4.13). For high-strength DOACs, no difference was found for ischaemic stroke compared with warfarin, however, there was a significant reduction in risk of bleeding events (HR $0.63,95 \% \mathrm{Cl} 0.44$ to 0.89 ) and death ( $\mathrm{HR} 0.40,95 \% \mathrm{Cl} 0.28$ to 0.58 ). Propensity score matching showed no difference in risk of ischaemic stroke or bleeding.

Conclusion We found that in the practice setting both DOAC formulations were similar to warfarin with regard to effectiveness and had no increased risk of bleeding.

\section{INTRODUCTION}

Direct oral anticoagulants (DOACs) were subsidised on the Pharmaceutical Benefits Scheme (PBS) in Australia in 2013 for prevention of stroke or systemic embolism
Strengths and limitations of this study

- A strength was the use of a sequential cohort approach to minimise channelling bias.

- A strength was that the results were stratified by direct oral anticoagulant (DOAC) strength.

- Unable to determine the dose of DOAC consumed by the patient.

- Indication for anticoagulant use was not recorded in our data.

in patients with atrial fibrillation (AF). At the time of listing, there was uncertainty around their cost-benefit advantage over warfarin, their potential bleeding risk and the limited strategies available to control overanticoagulation. ${ }^{1}$ Since listing, DOACs have gained significant market share with one-third of people commencing anticoagulant therapy initiated on a DOAC. ${ }^{2}$ This is partly because treatment with warfarin is complex due to anticoagulant response being highly variable between and within patients, and the requirement for regular monitoring to avoid overanticoagulation or therapeutic failure. DOACs offer an alternative treatment option, with fixed dosing based on clinical characteristics such as age, weight and renal function. However, there are concerns about the fixed dosing recommendations with DOACs, and the lack of the ability to monitor anticoagulation, particularly for the lower doses which are recommended for patients who typically have greater variability in drug concentrations and response. This concern is exacerbated by the relatively low proportion of patients in clinical trials ${ }^{3}$ who were on low-dose products for rivaroxaban and apixaban (22\% and $4.9 \%$ in rivaroxaban and apixaban trials, respectively).

We aimed to compare the effectiveness and safety of low-strength and highstrength formulations of DOACs to warfarin. 
Low-strength and high-strength products were analysed separately because different DOAC formulations are recommended for distinct patient subgroups and dose adjustment is required in patients at increased risk of bleeding.

\section{METHODS}

A balanced sequential cohort study design was employed to minimise channelling bias, a type of selection bias that may occur when a new medicine enters the market. ${ }^{4}$ Five cohorts representing sequential 4 monthly calendar intervals were established based on the date of initiation of oral anticoagulant therapy (index dispensing); cohort 1: patients initiated between 1 August 2013 and 30 November 2013, cohort 2: 1 December 2013 and 31 March 2014, cohort 3: 1 April 2014 and 31 July 2014, cohort 4: 1 August 2014 and 30 November 2014, cohort 5: 1 December 2014 and 31 March 2015.

The Australian Government Department of Veterans' Affairs (DVA) administrative claims database was used for this analysis. These data include all medicines dispensed under the PBS, and all medical, hospital and allied health services claimed by veterans. DVA maintains a client file including, date of birth, gender, date of death and family status for veterans, spouses and their dependents. Prescriptions are coded according to WHO's Anatomical, Therapeutic Chemical (ATC) classification system $^{5}$ and PBS item codes. ${ }^{6}$ Hospitalisations are coded according to International Classification of Disease, 10th Revision Australian Modification (ICD-10AM) codes. ${ }^{7}$

Patients were included if they initiated anticoagulation during the study period. Patients were considered as initiators if they had no dispensing of any oral anticoagulant in the 12 months prior to the index dispensing. Participants were included if they were 18 years or older, not in residential aged care and had DVA enrolment for at least 12 months prior to the index dispensing. DVA enrolment means that participants were eligible for all health services subsidised by DVA. Patients were excluded if they had an aortic valve procedure in the 12 months prior to initiating treatment (procedure codes: 3848800,3848801 , 3848900, 3848901, 3855301, 3855303, 3855601, 3855603, 3856201, 3856203, 3856501, 3856503).

Exposure to DOAC was identified using PBS item codes for the indication of AF (see online supplementary appendix A) and classified as either low-strength (apixaban $2.5 \mathrm{mg}$, dabigatran $110 \mathrm{mg}$, rivaroxaban $15 \mathrm{mg}$ ) or high-strength (apixaban $5 \mathrm{mg}$, dabigatran $150 \mathrm{mg}$, rivaroxaban $20 \mathrm{mg}$ ). Warfarin was identified using the ATC code B01AA03. ${ }^{8}$ Individuals in each cohort were followed for up to 12 months from their index dispensing or until they switched to another anticoagulant or died; whichever came first.

The primary effectiveness outcome was hospital admission for stroke (ischaemic or unspecified stroke) and the primary safety outcomes were hospitalisation for any bleeding event (gastrointestinal or other bleeding events) or haemorrhagic stroke (ICD10 codes provided in online supplementary appendix B). Secondary outcomes were hospitalisation for myocardial infarction (MI) or death.

An inverse probability of treatment weighting (IPTW) approach was used to compare the rate of outcomes separately for low-strength DOACs with warfarin and highstrength DOACs with warfarin. This method measures the average treatment effect (ATE), it is the average effect at the population level that would be seen if all individuals received the treatment compared with if none of these individuals received the treatment. Within each calendar cohort, logistic regression was used to estimate the probability, or propensity, of DOAC treatment compared with warfarin conditional on patient covariates measured at the time of treatment initiation. ${ }^{9}$ Separate propensity score (PS) models were created for high-strength DOAC compared with warfarin, and for low-strength DOAC compared with warfarin within each calendar cohort. Additionally, a separate PS model was built for each individual product. There were 31 covariates included in the PS models that were established a priori by clinicians as variables potentially associated with treatment selection and outcomes (table 1 and online supplementary appendix A). Each subject was weighted by the inverse of their probability of receiving the treatment that they actually received, calculated using the PS model. ${ }^{10}$ This process reweights the DOAC and warfarin treatment groups to create a pseudo population where the baseline covariates are independent of treatment. To reduce variance in treatment effects arising from individuals with large weights (PS near 0 or 1$),{ }^{11}$ weights were stabilised by multiplying the prevalence of DOAC treatment in participants dispensed a DOAC and one minus the prevalence of DOAC treatment in those dispensed warfarin. To limit the size of extreme weights, we then truncated the weights at the 5th and 95th percentile. For those, with weights below the 5 th and above the 95 th percentile, their weights were adjusted to those of the 5th or 95th percentiles. Balance in baseline covariates between DOAC and warfarin treatment groups, before and after weighting was compared using standardised differences using a $10 \%(0.1)$ threshold to signify imbalance. ${ }^{10} 12$

In the final analysis, a combined calendar cohort was created from cohorts 2-5. Cohort 1 was excluded as a Product Familiarisation Program (PFP) ${ }^{13}$ that provided company-sponsored access to dabigatran prior to PBS listing meant that we were unable to distinguish initiators of dabigatran from prevalent users from the PFP.

Cox proportional hazards model was used for all analyses.

We undertook a post hoc analysis for the secondary MI endpoint, where we stratified results by baseline aspirin use. A sensitivity analysis using PS matching was also undertaken, which measures the ATE, which is the effect on the outcome if individuals in the population were randomly assigned to treatment. Within each calendar cohort, DOAC initiators were matched on a 1:1 basis on the logit of their PS ${ }^{14}$ to their nearest neighbour initiated 


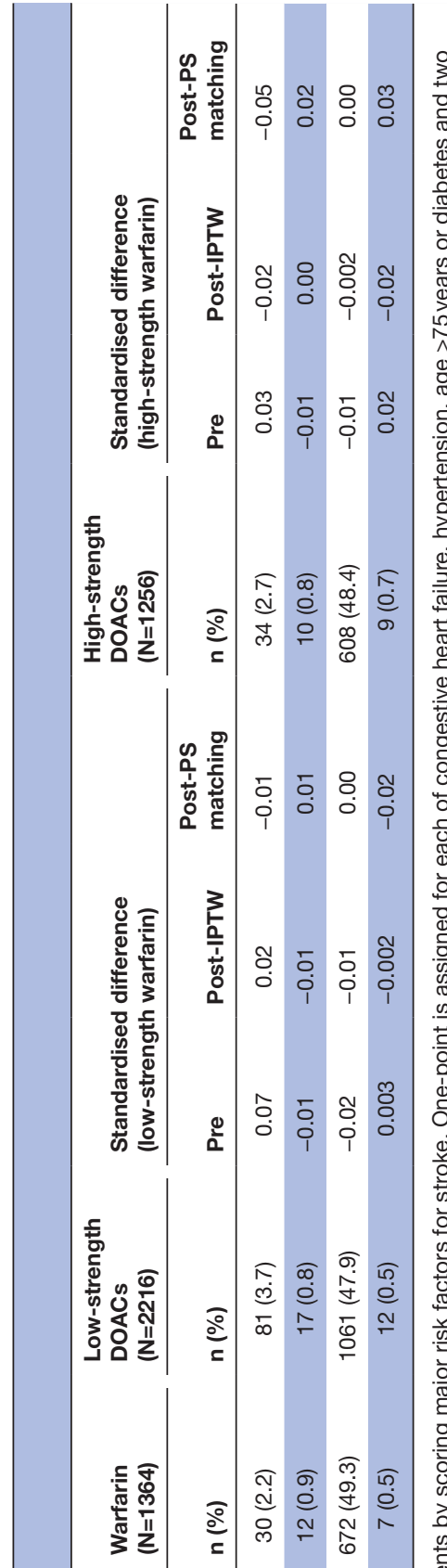

on warfarin, using a calliper width of 0.05 . Balance was measured using standardised differences between baseline covariates of the matched cohort. For each matched PS cohort, we applied a robust sandwich variance estimator to account for the matched design. ${ }^{15}$

All analyses were conducted using SAS V.9.4 (SAS).

\section{Patient and public involvement}

Patients and public were not directly involved in this research.

\section{RESULTS}

A detailed flow chart of the cohort selection process is presented in figure 1 . Table 1 details the baseline characteristics of patients by exposure group. Compared with those initiated on warfarin, patients initiated on low-strength DOACs were older, had a higher congestive heart failure, hypertension, Age ( $\geq 65=1$ point, $\geq 75=2$ points), diabetes, and stroke/TIA (2 points) $\left(\mathrm{CHADS}_{2}\right)$ score, fewer hospitalisations in the 60 days prior to initiation, lower prevalence of prior hospitalisation for ischaemic stroke, haemorrhagic stroke, MI and bleeding events and were more likely to be initiated treatment by a specialist. Compared with patients initiated on warfarin, patients initiated on high-strength DOACs were younger, had lower $\mathrm{CHADS}_{2}$ score, less hospitalisation in the 60 days prior to initiating therapy, lower prevalence of prior stroke or bleed and were more likely to be initiated treatment by a specialist. Based on standardised differences, for both the low-strength and high-strength groups, characteristics were more similar after weighting and PS matching compared with before (table 1). PS distributions before matching and PS matching are shown in figures 2 and 3, respectively.

No difference in risk of hospitalisation for stroke (ischaemic or unspecified) was found for low-strength DOACs or for individual products compared with warfarin (HR $1.00,95 \%$ CI 0.62 to 1.62 ) (table 2). Overall, no significant difference in risk of hospitalisation for bleeding or haemorrhagic stroke with low-strength DOACs compared with warfarin was found. There was a significantly lower risk of haemorrhagic stroke with low dose apixaban compared with warfarin (HR $0.17,95 \%$ CI 0.04 to 0.79 ).

No significant difference in risk of hospitalisation for stroke was observed between high-strength DOACs and warfarin (table 2). Compared with warfarin, a reduced risk of hospitalisation for bleeding was observed overall with high-strength DOACs (HR $0.63,95 \%$ CI 0.44 to 0.89 ) and individually, for high-strength apixaban (HR 0.33, $95 \%$ CI 0.17 to 0.65 ) but not rivaroxaban. No difference in risk of haemorrhagic stroke was observed with highstrength DOACS compared with warfarin.

For the secondary outcomes, there was no increased risk of MI for low-strength DOACs compared with warfarin, however, an increased risk was observed with low-strength apixaban compared with warfarin (HR 2.25, 95\% CI 1.23 
DVA participants ( $\geq 18$ years) with any prescription between Aug 2013 - March 2015 ( $\mathrm{N}=205663$ )

DVA participants with any oral anticoagulant (OAC) prescription $(\mathrm{N}=24892)$

\section{EXCLUDED}

DVA enrolment duration $<12$ months prior

to OAC prescription ( $\mathrm{N}=\mathbf{2 8 1 0}$ )

Residential aged care resident $(\mathrm{N}=4353)$

Prevalent OAC users in 12 months

preceding August 2013 ( $\mathrm{N}=14769$ )

OAC prescription for venous

thromboembolism prophylaxis following

hip or knee replacement ( $N=184)$

Aortic valve procedure in the prior 12

months ( $\mathrm{N}=159)$

Incident OAC users $(\mathrm{N}=6,894)$



Cohort 2-5 Incident OAC users $(\mathrm{N}=4,836)$

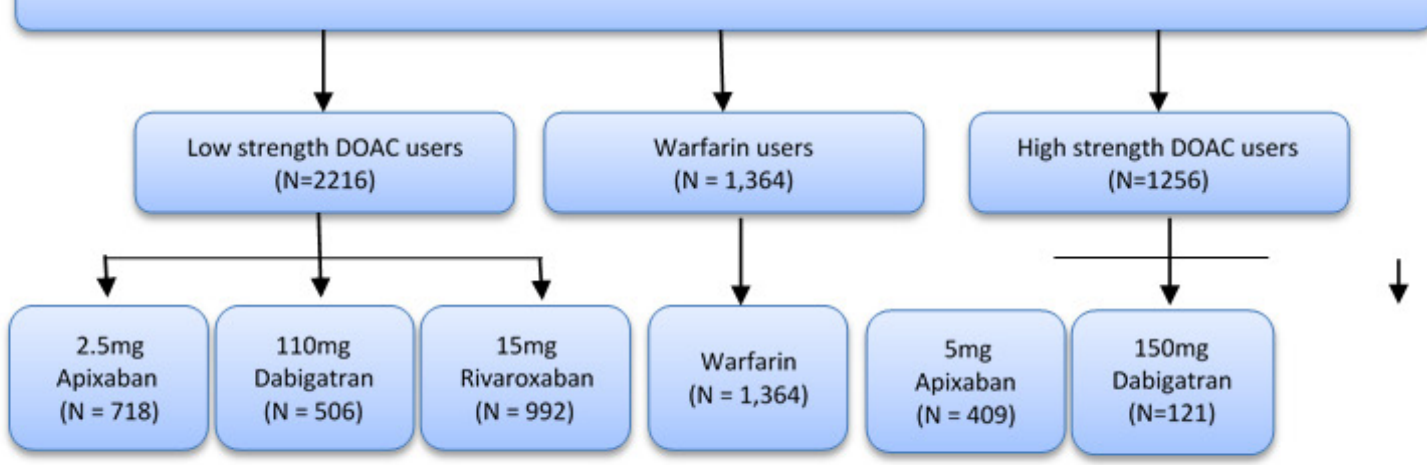

Propensity Matched analysis
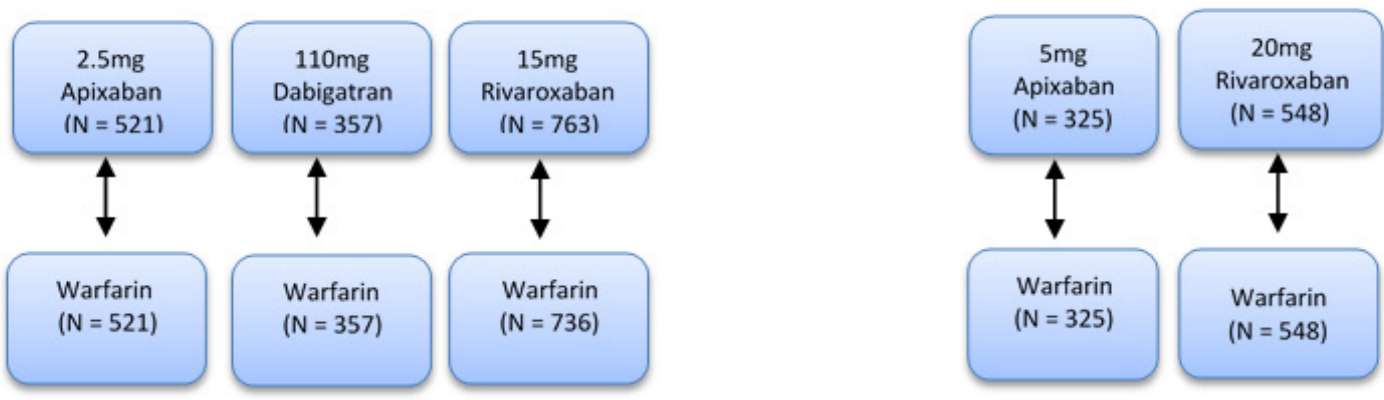

Figure 1 Flow chart of cohort selection. DOAC, direct oral anticoagulant; DVA, Department of Veterans' Affairs.

to 4.13) (table 3). There was a significant difference in the risk of death between low-strength DOACs and warfarin (HR 0.77 , 95\% CI 0.60 to 0.98 ), however, no difference for the individual DOACS was observed (table 3). For high-strength DOACs, no difference in risk of MI was observed compared with warfarin (table 3 ). The risk of death was significantly reduced overall with high-strength DOACs (HR 0.40, 95\% CI 0.28 to 0.58 ) and individually, 


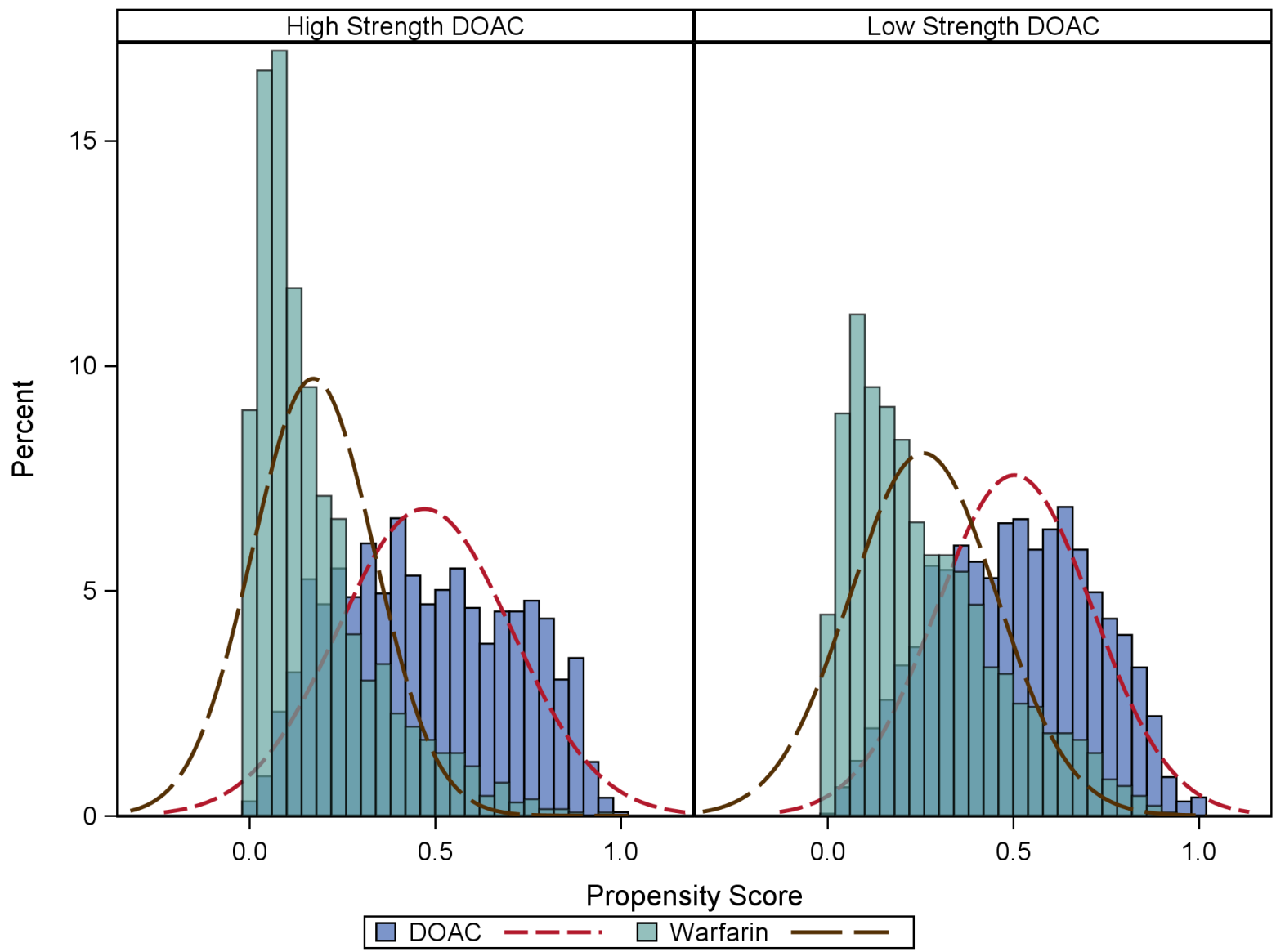

Figure 2 Density plots of the propensity score distribution for low-strength and high-strength DOACs prior to matching. DOAC, direct oral anticoagulant.

for apixaban (HR $0.25,95 \% \mathrm{CI} 0.11$ to 0.55$)$ and rivaroxaban (HR $0.60,95 \%$ CI 0.39 to 0.90 ).

In the post hoc analysis, a significantly increased risk of MI with low-strength apixaban compared with warfarin was found only in those not concomitantly dispensed aspirin at baseline (HR 2.31, 95\% CI 1.08 to 4.92). For those on aspirin at baseline, there was no significant difference in risk of MI between low-strength apixaban and warfarin (HR 2.12, 95\% CI 0.77 to 5.88) (data not shown).

Results of the PS-matched sensitivity analysis for the primary (table 4) and secondary endpoints (table 5) were consistent with those of the IPTW analysis. Comparisons of baseline covariates in the PS-matched cohort are described in online supplementary appendix table C.

\section{DISCUSSION}

Both low-strength and high-strength DOACs were similar to warfarin in terms of effectiveness, that is risk of ischaemic stroke. Risk of hospitalisation for bleeding was significantly reduced with high-strength DOACs compared with warfarin while no difference was observed for low-strength DOACs compared with warfarin. Our use of the IPTW and PS matching approaches meant that we were able to compare outcomes in patients initiated on low-strength or high-strength products to similar patients using warfarin. Stratification of results by DOAC strength is important as differences were observed between patients initiated on low-strength and high-strength DOACs, likely to be reflective of physicians' considerations of risk factors of both benefits and potential harms. Patients initiating low-strength DOACs were on average a decade older and had higher CHADS $_{2}$ scores than those on high-strength DOACs. These results suggest that recommendations for appropriate dosing appear to be adhered to in practice, and that patients at high risk of bleeding are likely prescribed low-strength DOACs rather than high strength. Our results need to be interpreted within the context of our study design, which was not intended to examine differences in outcomes specifically between high-strength and low-strength DOAC products, but rather, to compare the outcomes associated with high-strength or low-strength DOACs separately to similar patients who initiated warfarin.

Our results for high-strength DOACs are similar to a meta-analysis of randomised controlled trials (RCT) which found a reduced risk of ischaemic stroke and a lower risk of mortality for DOACs compared with warfarin; however, this meta-analysis did not differentiate between low-strength and high-strength products or the risk of 


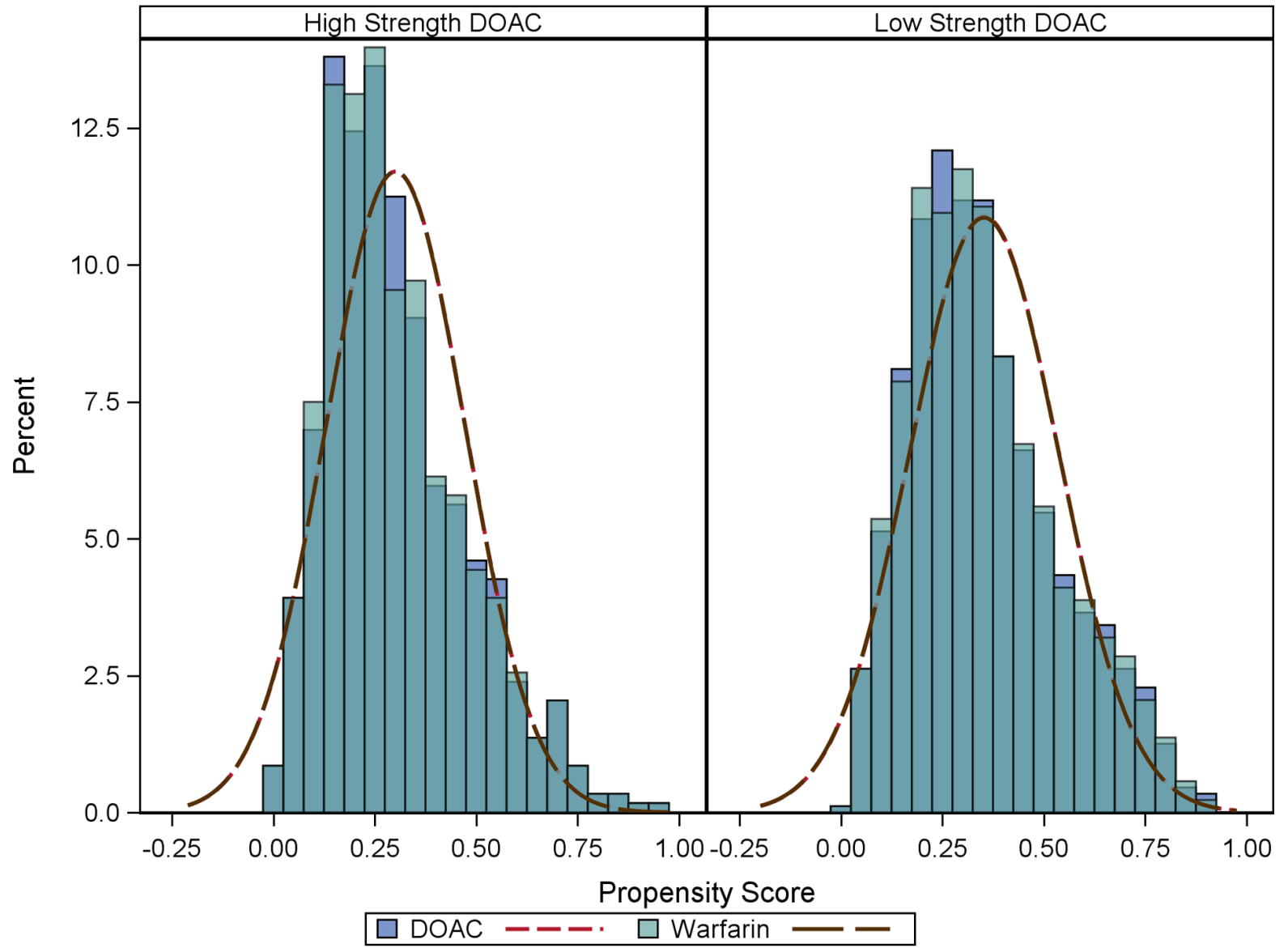

Figure 3 Density plots of the propensity score distribution for low-strength and high-strength DOACS after matching. DOAC, direct oral anticoagulant.

bleeding with different strengths. ${ }^{16}$ A study comparing results of 10 observational studies using PS adjustment with results from five RCTs found no difference in the estimates between RCTs and observational studies. This study found RCT and observation study results were similar with both finding a significantly reduced risk of ischaemic stroke with DOACs and no difference in the risk of major bleed. ${ }^{17}$ Neither of these studies examined outcomes of DOACs with warfarin separately for high-strngth and low-strength products. A Danish observational study investigating low-strength formulations ${ }^{18}$ found no difference in the risk of ischaemic stroke or bleeding between the low-strength DOACs and warfarin. An analysis of two UK primary care databases ${ }^{19}$ found no difference in the risk of ischaemic stroke with lower dose products but found a reduced risk of major bleed with lower dose apixaban. Additionally, in that study, ${ }^{19}$ higher doses of apixaban were associated with lower risk of major bleed with no difference in risk of ischaemic stroke compared with warfarin. In our study, we found no difference in risk of hospitalisation for ischaemic stroke or bleeding with low-strength DOACs. These data suggest that the benefits of DOACs demonstrated in RCTs, where the majority of patients were prescribed the higher doses, may not be applicable in real-life use, where a larger number of patients are suitable for the lower doses.
Our result of an increased risk of MI for low-strength apixaban should be examined further. Our post hoc analysis suggests it is possible that the concomitant use of other antiplatelet medicines such as aspirin may contribute to observed differences in risks. Use of aspirin at baseline was similar across our study treatment groups with over one-third of all patients using concurrent aspirin, however, an significantly increased risk of MI was only found with low-dose apixaban in those patients who were not concomitantly dispensed aspirin. Evidence of the efficacy of low-dose apixaban is not well established, with only $4.9 \%$ of subjects (428 subjects) randomised to low-dose apixaban in clinical trials. ${ }^{3}$ In the absence of concomitant antiplatelet treatment low-dose apixaban may be insufficient to protect against the risk of MI.

A strength of our study is our use of a sequential cohort approach with cohorts determined by 4-month calendar periods. This approach was used to minimise channelling bias, in which early initiators of DOACs were very different to warfarin initiators in the same period and a PS model was estimated in each calendar cohort to ensure comparability between treatment groups within each calendar period. ${ }^{40}$ We used an IPTW approach to determine ATE, reflecting the average effect at the population level. Weighting creates a pseudo population where the baseline covariates are independent of treatment. 

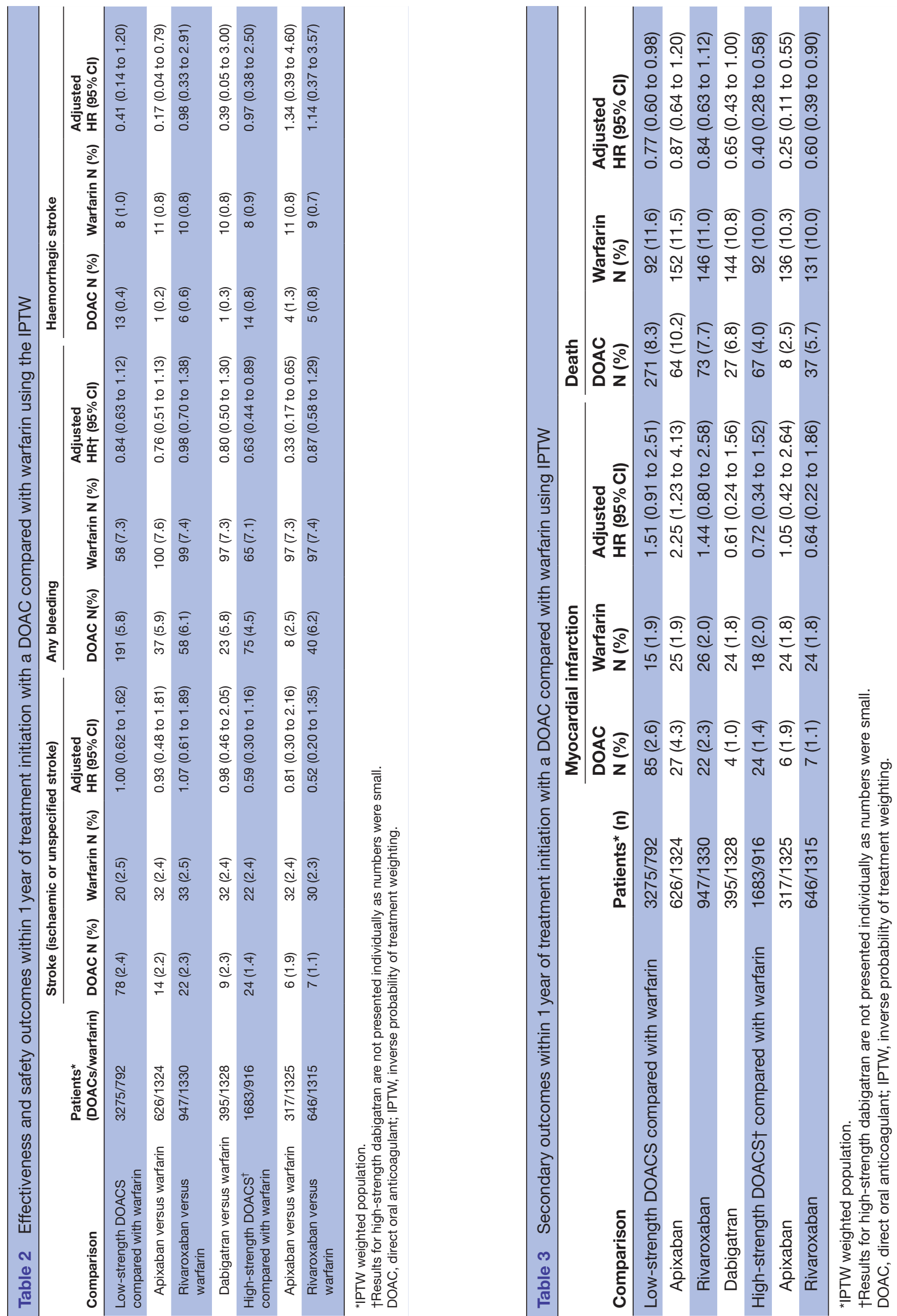

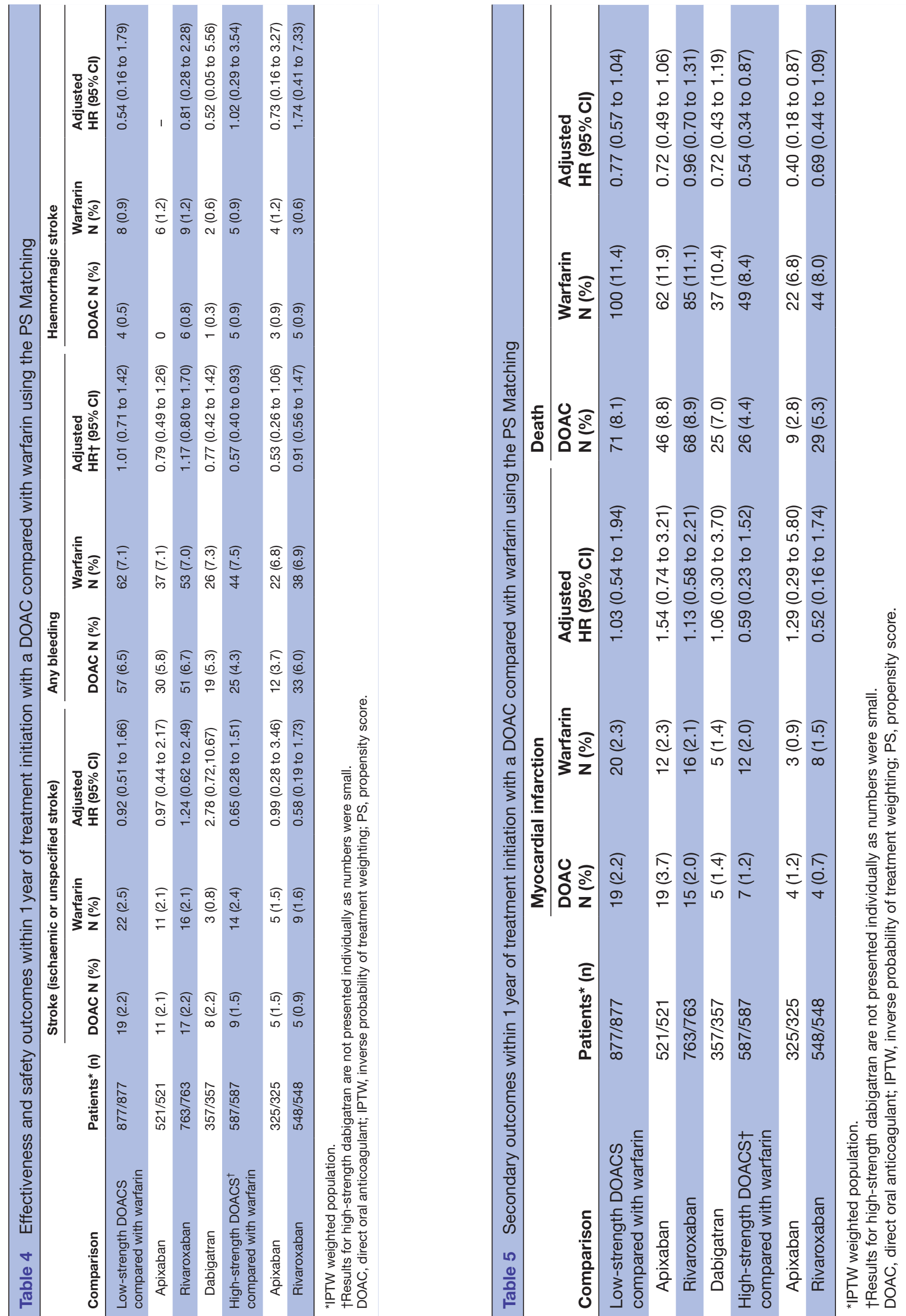
Additionally, PS matching was used as a sensitivity analysis finding similar results with the exception of bleeding risk, which was found to be lower with high-strength DOACs in the weighted analysis but not different in the matched analysis. Regardless of the method used to account for confounding, we did not observe a significantly increased risk of bleeding with DOACs compared with warfarin.

A limitation of our analysis was that we were unable to determine the dose of DOAC consumed by the patient as the data only contain information on the strength dispensed. Because of the fixed dosing recommendations for DOACS, the strengths dispensed likely to reflect the doses consumed. Additionally, we were unable to determine the indication for anticoagulant use. To minimise misclassification, we excluded patients with a recent hip or knee replacement surgery who may have been treated with DOACS for deep-vein thrombosis (DVT) prophylaxis. We were unable to specifically exclude use of DOACs for treatment of DVT. We identified our outcome events through administrative hospital claims, therefore, we were unable to validate our outcomes as we do not have access to hospital records. Another limitation is that variables used in the PS estimation were restricted to those available in the data source hence some residual confounding may remain.

Our study, performed in the Australian veteran population, is likely to be generalisable to the older population with $\mathrm{AF}$, however, may not be generalisable to other healthcare settings and patient populations. Previous research has reported that there was no difference in use of practitioners, health services and treatment between veteran and non-veteran patients in both the primary and tertiary Australian care sectors after adjustment for age, service-related disability and marital status. ${ }^{21}$ We focused on incident users of anticoagulants and those with prior warfarin use were excluded. Further research should examine the safety and effectiveness of DOACs in populations switching from warfarin. The limited use of highstrength dabigatran meant we were unable to determine outcomes of this treatment. Additionally, a number of hospitalisation outcome events were small, particularly for haemorrhagic stroke, and consequently confidence intervals were wide. Ongoing monitoring of the safety of DOACs will be required as larger populations are exposed to these medicines.

In conclusion, our analysis of patients initiated on anticoagulant treatment, indicated that both high-strength and low-strength DOACs were similarly effective as warfarin in the prevention of stroke. High-strength DOACs were associated with a reduced bleeding risk, however, this appeared to be limited to high-strength apixaban, which is consistent with clinical trial evidence. Our significantly reduced risk of hospitalisation for bleeding with highstrength products is likely explained by appropriate selection of lower risk patients for the high-strength products. The similar rate of bleeding events with low-strength DOACS and warfarin likely reflects the high baseline risk of bleeds in those indicated for low-strength treatment.
Our finding of an increased risk of MI for low-strength apixaban requires further exploration.

\section{Author affiliations}

${ }^{1}$ School of Pharmacy and Medical Sciences, Quality Use of Medicines and Pharmacy Research Centre, University of South Australia, Adelaide, South Australia, Australia

${ }^{2}$ Sansom Institute for Health Research, University of South Australia, Adelaide, South Australia, Australia

${ }^{3}$ Discipline of Pharmacology, University of Adelaide, Adelaide, South Australia, Australia

${ }^{4}$ Department of Clinical Pharmacology, Royal Adelaide Hospital, Adelaide, South Australia, Australia

Acknowledgements We acknowledge the support of the data custodian, the Australian Government Department of Veterans' Affairs (DVA) which provided data for the conduct of this study.

Contributors EER, SS, NLP, LMKE and GC contributed to the conception and design of this study. ER and MK carried out the analysis and NLP provided statistical advice. NLP and KD drafted the manuscript. SS, GC, LMKE and EER critically revised the manuscript for important intellectual content. All authors took part in interpreting the data for this study. All authors commented on and helped to revise drafts of this paper. All authors have approved the final version.

Funding The work was supported by an Australian Government National Health and Medical Research Council (NHMRC) Centre of Research Excellence in PostMarketing Surveillance of Medicines and Medical Devices grant (APP1040938). EER is supported by NHMRC APP1110139.

Competing interests All authors have completed the Unified Competing Interest form (available on request from the corresponding author).

Patient consent for publication Not required.

Provenance and peer review Not commissioned; externally peer reviewed.

Data sharing statement № additional data are available. The data that support the findings of this study are available from the Australian Government Department of Veterans' Affairs but restrictions apply to the availability of these data, which were used under licence for the current study, and so are not publicly available.

Open access This is an open access article distributed in accordance with the Creative Commons Attribution Non Commercial (CC BY-NC 4.0) license, which permits others to distribute, remix, adapt, build upon this work non-commercially, and license their derivative works on different terms, provided the original work is properly cited, appropriate credit is given, any changes made indicated, and the use is non-commercial. See: http://creativecommons.org/licenses/by-nc/4.0/.

\section{REFERENCES}

1. Ageing ADoHa. Issues and Options Paper: Review of Anticoagulation Therapies in Atrial Fibrillation. 2012.

2. Hripcsak G, Ryan PB, Duke JD, et al. Characterizing treatment pathways at scale using the OHDSI network: Proceedings of the National Academy of Sciences of the United States of America, 2016;113:7329-36.

3. Gómez-Outes A, Terleira-Fernández Al, Calvo-Rojas G, et al. Dabigatran, Rivaroxaban, or Apixaban versus Warfarin in patients with nonvalvular atrial fibrillation: a systematic review and metaanalysis of subgroups. Thrombosis 2013;2013:640723-18.

4. Schneeweiss S, Gagne JJ, Patrick AR, et al. Comparative efficacy and safety of new oral anticoagulants in patients with atrial fibrillation. Circ Cardiovasc Qual Outcomes 2012;5:480-6.

5. World Health Organization (WHO). Collaborating Centre for Drug Statistics Methodology. Guidelines for ATC classification and DDD assignment. Oslo: WHO, 2014.

6. Australian Government Department of Health. Pharmaceutical Benefits Services. Canberra: Australian Government (Department of Health), 2016.

7. Australian Consortium for Classification Development (ACCD). The International Statistical Classification of Diseases and Related Health Problems, tenth revision, Australian modification (ICD-10-AM/ACHI) ACS). 10th edn. Darlinghurst: Independent Hospital Pricing Authority, 2017.

8. Australian Medicines Handbook. Anticoagulants. In: Rossi S, (ed). 2016 Australian Medicines Handbook. Adelaide: Australian Medicines Handbook Pty. Ltd.; 2016:325. 
9. Austin PC, Grootendorst P, Anderson GM. A comparison of the ability of different propensity score models to balance measured variables between treated and untreated subjects: a Monte Carlo study. Stat Med 2007:26:734-53.

10. Austin PC. An introduction to propensity score methods for reducing the effects of confounding in observational studies. Multivariate Behav Res 2011;46:399-424.

11. Cole SR, Hernán MA. Constructing inverse probability weights for marginal structural models. Am J Epidemiol 2008;168:656-64.

12. Normand ST, Landrum MB, Guadagnoli E, et al. Validating recommendations for coronary angiography following acute myocardial infarction in the elderly: a matched analysis using propensity scores. J Clin Epidemiol 2001;54:387-98.

13. Attia JR, Pearce R. The use, misuse and abuse of dabigatran. Med $J$ Aust 2013;198:356-7.

14. Rosenbaum P, Rubin D. Constructing a control group using multivariate matched sampling methods that incorporate the propensity score. Am Stat 1985;39:33-8.

15. Gayat E, Resche-Rigon M, Mary JY, et al. Propensity score applied to survival data analysis through proportional hazards models: a Monte Carlo study. Pharm Stat 2012;11:222-9.

16. Miller CS, Grandi SM, Shimony A, et al. Meta-analysis of efficacy and safety of new oral anticoagulants (dabigatran, rivaroxaban, apixaban) versus warfarin in patients with atrial fibrillation. Am J Cardiol 2012;110:453-60.
17. Li G, Holbrook A, Jin Y, et al. Comparison of treatment effect estimates of non-vitamin $\mathrm{K}$ antagonist oral anticoagulants versus warfarin between observational studies using propensity score methods and randomized controlled trials. Eur J Epidemiol 2016;31:541-61.

18. Nielsen PB, Skjøth F, Søgaard M, et al. Effectiveness and safety of reduced dose non-vitamin $\mathrm{K}$ antagonist oral anticoagulants and warfarin in patients with atrial fibrillation: propensity weighted nationwide cohort study. BMJ 2017;356:j510.

19. Vinogradova Y, Coupland C, Hill T, et al. Risks and benefits of direct oral anticoagulants versus warfarin in a real world setting: cohort study in primary care. BMJ 2018;362:k2505.

20. Schneeweiss S, Gagne JJ, Glynn RJ, et al. Assessing the comparative effectiveness of newly marketed medications: methodological challenges and implications for drug development. Clin Pharmacol Ther 2011;90:777-90.

21. Australian Institute of Health and Welfare. A profile of Australia's veterans 2018. Cat. no. PHE 235. Canberra: AlHW, 2018.

22. Gage BF, Waterman AD, Shannon W, et al. Validation of clinical classification schemes for predicting stroke: results from the National Registry of Atrial Fibrillation. JAMA 2001;285:2864-70.

23. Pratt NL, Kerr M, Barratt JD, et al. The validity of the Rx-Risk Comorbidity Index using medicines mapped to the Anatomical Therapeutic Chemical (ATC) Classification System. BMJ Open 2018;8:e021122. 